\title{
Case Report-A Case of Peripartum Cardiomyopathy
}

\author{
Authors
}

\section{Dr Sarang Videkar, Dr R.P.Mundle, Dr P.K.Deshpande, Dr Dhoble}

NKP Salve Institute of Medical Sciences and Research Centre and Lata Mangeshkar Hospital

\begin{abstract}
PERIPRTUM CARDIOMYOPATHY was 1st described in the 18th century.

1971 Demakis et al described 27 patients, who presented during the purperium with cardiomegaly, abnormal ECG findings and congestive cardiac failure.Hence they named the syndrome "The peripartum cardiomyopathy".

It is a rare type of cardiomyopathy associated with significant mortality and morbidity as well as heart failure in late pregnancy or purperium.

Incidence-1:2000 to 1:15000.

This case is a classic example of how to manage heart failure in pregnancy and and features of cardiomyopathy as well as heart failure can mimic ARDS.

This case highlights risk factors, etiology, investigations, management options, newer treatment modalities for peripartum cardiomyopathy.

Beta blockers, ACE inhibitors, diuretics, nitrates are main stay of treatment.
\end{abstract}

A 24 Yr. patient $\mathrm{G}_{3} \mathrm{P}_{2} \mathrm{~L}_{2}$ with twin pregnancy 38 weeks, brought by relatives in emergency department with Complaints of

1. Dry cough and enema feet since 15 days

2. Progressive breathlessness since 4 days

3. Reduced level of consciousness since 1 day.

Patient was admitted in Obstetrics ICU. Medicine opinion was taken in view of respiratory distress.

\section{On Examination}

- Patient was drowsy, not obeying simple verbal commands.

- Patient was dyspnoeic, RR-38/min,P150/min,BP-160/100 mm Hg ,JVP was raised ,bilateral pedal oedema was present ,had bilateral coarse crepitations. $\mathrm{SpO}_{2}$ was $88 \%$ on room air and $90 \%$ with 2 lit. $\mathrm{O}_{2}$,

- Heart sounds were normal and plantars were bilateral flexors.

- Fundus-Grade 1 hypertensive retinopathy, but no papilledema.

- $\mathrm{ABG}$-PH-7.14, $\mathrm{PO}_{2}$ 73, $\mathrm{PCO}_{2} 38.8, \mathrm{HCO}_{3}$ 21.2 and $\mathrm{SO}_{2} \quad 89 \%$ suggesting severe metabolic acidosis.

- Hence urgent intubation and ventilator support was advised.

- Patient was stabilised and then LSCS was done.

- Patient was kept on ventilator and was gradually weaned off and finally extubated after 3 days. 
- Patient was then transferred to medicine ICU as there was no Obstetrics management and patient was in heart failure even after delivery.

- While in ICU, patient had irrelevant talk. Expert PSYCHIATRIST opinion was taken and it was diagnosed as Postpartum psychosis with delirium.

\section{Past History}

- History of PIH was present; patient was taking Tab. Alphadopa $250 \mathrm{mg}$ BDsince 2 months on.

- Her previous 2 pregnancies were Full Term Normal Delivery; history of PIH was present with both of these pregnancies.

- No H/O fever, burning micturition.

- No h/o jaundice, blood transfusion, haemoptysis.

- No h/o convulsions, oliguria, blurring of vision.

- No

$\mathrm{H} / \mathrm{O}$

diabetes/ tuberculosis/asthma/thyroid disease.

\section{Investigations}

ABG -PH-7.14, $\mathrm{PO}_{2} 73, \mathrm{PCO}_{2} 38.8, \mathrm{HCO}_{3} 21.2$ and $\mathrm{SO}_{2} 89 \%$ suggesting severe metabolic acidosis.

X-RAY Chest(lead apron guarded)-Pulmonary oedema with Cardiomegaly.

Other tests-

VDRL/HIV/HBSAG-Negative,

Sickling test-Negative,

Urine Exam-Normal,

TSH-Normal,

LFT-Normal, Sr.Alb-3.0,Total prot-4.6,KFTNormal,

Hb-11.3gm \%, TLC-14000,Platlets-1.59lacs $/ \mathrm{mm}^{3}$.

2-D ECHO-done in MEDICINE ICU,3 days after delivery -

LVEF 15\%,no valve lesion, global hypokinesia, IVC- dilated, mild pericardial effusion, PERIPARTUM CARDIOMYOPATHY.

Repeat 2-D ECHO-after one and half months, when patient came for follow up-
LVEF 67\%, no RWMA,no pericardial effusion and IVC-normal.
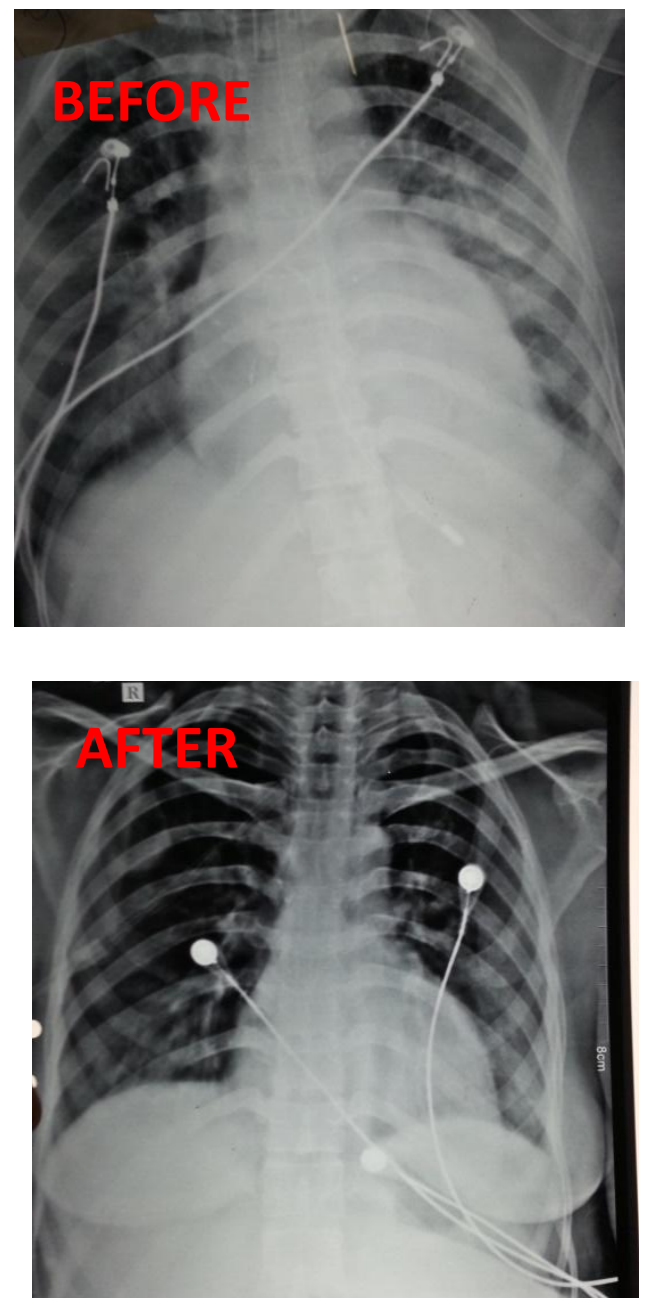

\section{Treatmentoffered To The Patient}

- Patient was treated with---

- Antibiotics- Inj.Pipracillin and Tazobactum 4.5 gm. TDS, Inj. Metronidazole $500 \mathrm{mg}$ TDS,

- Diuretics- Inj. Lasix 40 mg BD

- ACE inhibitors -T .Ramipril 5mg BD (lactation was stopped during ACEI therapy)

- Beta blockers- T.Met-XL 25 mg BD

- Anti coagulantsInj.Heparin(conventional)5000 IU SC BD.

- Also with T.Risdone $0.5 \mathrm{mg} \mathrm{BD}$ and T.Lopez $1 \mathrm{mg}$ BD for 15 days and patient was recovered from psychosis and delirium. 


\section{Review Of Literature}

- Periprtum Cardiomyopathy was $1^{\text {st }}$ described in the 18th century.

- 1971 Demakis et al described 27 patients, who presented during the purperium with cardiomegaly, abnormal ECG findings and congestive cardiac failure.

- Hence they named the syndrome "The peripartum cardiomyopathy".

- It is a rare type of cardiomyopathy associated with significant mortality and morbidity as well as heart failure in late pregnancy or purperium.

- Incidence-1:2000 to 1:15000.

\section{Definition}

- 3 clinical and 1 ECHO criteria-

- Development of heart failure during last trimester of pregnancy or $1^{\text {st }} 6$ months post partum.

- Absence of any identifiable cause for cardiac failure.

- Absence of any recognizable heart disease prior to last trimester of pregnancy.

- 2-D ECHO-

- Ejection fraction <45\%,Left ventricular fractional shortening less than $30 \%$ or left ventricular end-diastolic dimension $>2.7 \mathrm{~cm} / \mathrm{m}$ square of body surface area.

\section{Risk Factors}

- Higher maternal age

- Multipara-previous exposure may increase immune response

- Multifoetal pregnancy(2/3)

- $\mathrm{PIH}$

- Obesity

- Maternal cocaine and alcohol abuse, Smoking

- Long term tocolytic therapy

- Selenium deficiency-its anti oxidant

- GBS

- Myocarditis
Etiology

1. Nutritional-salt overload.

2. A/W myocarditis-7 to $78 \%-\mathrm{a} / \mathrm{w}$ HSV, EBV and CMV----cross reactivity of antiuterine antibodies against cardiac muscles.

3. Chimerism-mixture of genotypesimmunological response

4. Apoptosis and inflmm-incr CRP and TNF alpha, increased proteolytic cathepsin D activity in cardiomyocyte result in $16 \mathrm{kDa}$ prolactin fragment with anti-angiogenic and apoptotic properties.

5. Abn.Hemodynamics- rise in blood vol and Cardiac output.

6. Unmasking of latent idiopathic dilated cardiomyopathy by preg.

\section{Clinical Features}

Symptoms-

- Cough, orthopnoea, PND, fatigue, palpitations, weight gain, haemoptysis ,chest pain \& abdominal pain, altered mental status, psychiatric symptoms.

- Signs-

- Cardiomegaly, tachycardia, decr $\mathrm{SPO}_{2}$, elevated JVP,S3 gallop and loud P2,MR or TR and pulmonary crepitations.

- Peripheral oedema, ascites and hepatomegaly.

- Arrhythmias-May cause emboli.

- Pericardial effusion, proteinuria, preeclampsia.

\section{Diagnosis}

- We should consider PPCMP in any peripartum patient with signs and symptoms of heart failure.

\section{Investigations}

- X-Ray-guarded

- ECG

- 2D ECHO-May show reduced LVEF,LVH,MR/TR/PR.....review ECHO be done after 6 weeks, 3 months and 6 months 
- Endomyocardial Biopsy-myocarditis

- Viral and bacterial titre and cultures

- MRI-Myocarditis has a nonvascular distribution in the subepicardium with a nodular or band-like pattern, whereas ischemia has a vascular distribution in a sub-endocardial or trans mural location.

- Coronary angiography,Right heart catheterization.

- Biochemical evaluation-CK MB,TROPONIN-T.

\section{Differencial Diagnosis}

1.Idiopathic Dilated Cardiomyopathy (IDCM)-

Differences between PPCM and IDCM

1. PPCM-younger age and better prognosis.

2. Incidence-PPCM-higher.

3. PPCM mc in postpartum (78 - 93\%,IDCM usually 2 nd trimester.)

4. PPCM exclusively affects pregnancy and recurrent PPCM is seen in the peripartum period.

5. Varying types of hemodynamic patterns are seen in PPCM.

6. Unique sets of antigen and antibodies against myocardium-seen in PPCM compared to IDCM patients.

7. Incidence of myocarditis-higher in PPCM.

8. Heart size returns to normal after delivery in PPCM.

9. Contrary to IDCM,PPCM may lead to rapid worsening of clinical condition.

2.Acute MI

3.Valvular Heart Ds-MS,MR

4.Pulmonary Thromboembolism

5.Severe PIH

\section{Management}

- Low Na diet, fluid restriction <2 lit/day.

- During pregnancy-ICUif needed,BP,CVP monitoring

- $\mathrm{ACE}$ and ARBs are C/I in pregnancy.

- Digoxin, loop diuretics, Na restriction and drugs reducing afterload such as hydralazine and nitrates.
- Amlodipine - improves survival in non ischemic CMP.

- During Post Partum period

- ACE Inhibitors- T.Enalapril(max.) 20 mg/day and ARBs are useful

- Diuretics, Dioxin, BB - Metoprolol/ Carvedilol.

- Anticoagulation candidates- in bedridden patients, if $\mathrm{LVEF}<35 \%$, atrial fibrillation, mural thrombi, obese patients and with history of thromboembolism . Heparinante partum period and heparin or warfarin in the post partum period, as warfarin- $\mathrm{c} / \mathrm{I}$ pregnancy.

\section{Newer Options}

- Pentoxifylline-inhibits TNF-alpha,400 mg TDS.

- Role of Bromocriptine and Cabergoline-as prolactin increases risk of PPCMP.

- Immune modulators-IV IG., plasmapheresis,

- Other drugs-CCB, statins, monoclonal AB and INF-beta.

- Other Interventions and Devices -an intraaortic balloon pump or LV assist devices.

- Rare-continuous veno-venous haemodialysis

- Inodilators such as amrinone, milrinone, enoximone and levosimendan.

- Levosimendan-helps in weaning of inotropes in inotrope -dependent cases.

- Nesiritide, BNP, reduce elevated PCWP and lead to clinical improvement.

- Last option-Cardiac transplantation.

Prognosis

Nearly $50 \%$ patients will normalize their LVEF during follow up within 6 months.

Prognosis is directly correlated to recovery of LVEF.

15 to $20 \%$ patients do not show improvement in LVEF at the end of 6 months. 
THINKING OF NEXT PREGNANCY IN PATIENT OF DCM

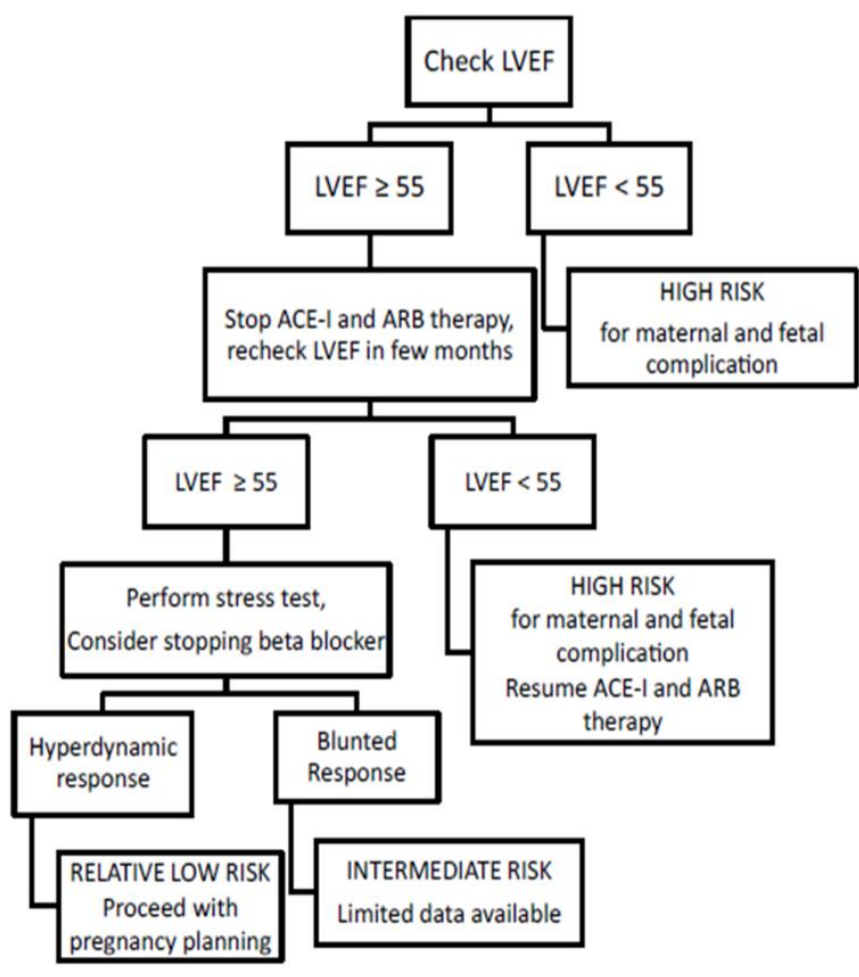

\section{Reference}

1. Selle T, Renger I, Labidi $\mathrm{S}$ et al; Reviewing peripartum Cardiomyopathy; current state of knowledge. Future Cardiol 2009;5:175-89.

2. Pandit V, Shetty S, Kumar A et al; Incidence and out come of peripartum Cardiomyopathy from a tertiary hospitalin South India. Trop Doct 2009;39:168-9.

3. Desai D, Moodley J, Naidoo D; Peripartum Cardiomyopathy Experience at king Edward VIII Hosp, Durban, South Africa and a review of the literature. Trop Doct 1995;25:118 23.

4. Fett JD, Christie LJ, Carraway RD et al; Five year prospective study of the incidence and prognosis of the peripartum cardiomyopathy at a single institution. Mayo Clinic Proc 2005;80:1602-6.

5. Sanderson JE,Adesanya CO, Anjorin FIet al; Postpartum cardiac failure- heart failure due to volume over load? Am Heart J 1979;97:613-621.
6. Shamiri MQ, Nozha MM; Peripartum cardiomyopathy: Searching for a better understanding. Saudi Med J 2003;24:104851.

7. ummala PP, Rao KS, Akhter MW et al; Peripartum cardiomyopathy. Clinical profile of 100 patients diagnosed in the United States. Circulation 1999;100: I579..

8. Demakis JG, Rahimtoola SH; Peripartum Cardiomyopathy.

Circulation 1971;44;964-68.

9. Cenac A, Simonoff M, Moretto P et al; Low plasma selenium is a risk factor for peripartum cardiomyopathy. A comparative study in Sahelian Africa. Int $\mathbf{J}$ Cardiol 1992;36:57-9.

10. Rizeg MN, Rickembacher PR, Fowler MB et al; Incidence of myocarditis in peripartum cardiomyopathy. Am J Cardiol 1994;74:474.

11. Felker GM, Thomson R,Hare JM et al. Underlying causes and long term survival in patients with initially unexplained cardiomyopathy. N Engl J Med 2000;342:1077-84. 